\title{
Helicobacter Pylori Infection Is Associated with Dyslipidemia and Increased Levels of Oxidized LDL in Type-2 Diabetes Mellitus
}

\author{
Mohamed Hasan Mukhtar ${ }^{1}$, Wesam Ahmed Nasif ${ }^{1,2}$, Abdullatif Taha Babakr ${ }^{1 *}$ \\ ${ }^{1}$ Department of Medical Biochemistry, Faculty of Medicine, Umm Al-Qura University, Makkah, KSA \\ ${ }^{2}$ Molecular Biology Department, Genetic Engineering and Biotechnology Research Institute, Sadat City \\ University, Sadat City, Egypt \\ Email:mhmukhtar@uqu.edu.sa,wnasif2003@yahoo.com, "atbabakr@uqu.edu.sa
}

Received 9 June 2016; accepted 9 July 2016; published 12 July 2016

Copyright (C) 2016 by authors and Scientific Research Publishing Inc.

This work is licensed under the Creative Commons Attribution International License (CC BY).

http://creativecommons.org/licenses/by/4.0/

(c) (i) Open Access

\section{Abstract}

Emerging data now indicate and address the strong relationship between $H$. pylori infection and the incidence of Type $2 \mathrm{DM}$, a growing body of evidence suggests that the infection with $H$. pylori may be associated with insulin resistance, chronic inflammation, long-term diabetes complications, and cardiovascular risk factors. The present study was conducted to evaluate the relationship between the infection with Helicobacter pylori and disturbance in Lipid profile in Type 2 Diabetic patients. One hundred and five participants were enrolled, categorized into two groups of $H$. pylori positive cases and negative controls according to their results of $H$. pylori IgG antibodies. Subjects in both groups fill the structured questionnaire. Blood samples were drawn for measuring the FBS, $2 \mathrm{hr}$-PP blood sugar, HbA1c, Lipid profile and oxidized LDL. The obtained results were statistically analyzed. The study methodology was approved by the Biomedical Ethics Committee in the Faculty of Medicine, Umm Al-Qura University, Makkah, KSA. 48 cases (45.7\%) were diagnosed as $H$. pylori seropositive and $57(54.3 \%)$ were negative. There is no significant difference in the mean age or mean BMI between the $H$. pylori negative and positive cases. Glycemic control was similar in the two groups. Total Cholesterol was higher in cases of positive $H$. pylori compared to negative controls $(P<0.001)$ and Triglycerides was significantly elevated too $(P<0.005)$. No significant difference in the levels of HDL-Cholesterol between the two groups, while the mean LDL-Cholesterol was found to be significantly increased in cases of $H$. pylori positive compared to negative controls $(P<0.001)$. Oxidized LDL levels in the positive cases was found to be increased significantly $(P=0.001)$ compared to negative controls. Infection with $H$. pylori is associated with increased levels of Total Cholesterol, Triglycerides, LDL-C and oxidized LDL in Type 2 Diabetic patients.

\footnotetext{
${ }^{*}$ Corresponding author.
} 
Keywords

\section{H. pylori, Diabetes, Oxidized LDL}

\section{Introduction}

Helicobacter pylori is an spiral-shaped gram-negative pathogenic bacterium that specifically colonizes in the gastric epithelium and causes several acute and chronic gastro-duodenal diseases including chronic gastritis, peptic ulcer disease, and/or gastric malignancies [1]. It is estimated that half of the world population was infected with $H$. pylori, with more prevalence in developing countries [2] [3].

Eradication of $H$. pylori was reported to lead to a more healthy development in young population. In patients with unexplained iron deficiency anemia (IDA), successful eradication of $H$. pylori infection leads to improvement in hemoglobin, iron, total iron-binding capacity and ferritin values [4].

Recent studies indicate a strong association between $H$. pylori infection and the incidence of Type 2 diabetes mellitus [5], a growing body of evidence suggests that the infection with $H$. pylori may cause insulin resistance and chronic inflammation related to diabetes [6]. The mechanisms of pathogenesis of diabetes are complex, including insulin resistance and chronic inflammation, moreover, previous studies suggest a correlation between $H$. pylori infection and poor glycemic control [7].

The extra-intestinal manifestations of $H$. pylori have been shown in many previous studies [8], considerable evidence suggest the potential role of Helicobacter pylori (H. pylori) infections in the initiation, development or persistence of atherosclerosis and coronary heart disease (CHD) [1] [9] [10].

There remains a critical need to better understand the underlying disease mechanisms responsible for diabetes complications, which may help in developing new and improved therapeutic strategies. Atherosclerosis and subsequent events are considered as the most prevalent and serious complications of diabetes mellitus [11], dyslipidemia and oxidized low density lipoproteins (ox-LDL) play a major role in macrovascular complications of diabetes mellitus, and $H$. pylori was considered to be a reversible risk factor in Coronary Artery Disease (CAD) [12].

The present study was conducted to investigate the possible relationship between $H$. pylori infection and disturbance in lipid profile, especially the levels of atherogenic ox-LDL in Type-2 diabetic patients.

\section{Subjects and Methods}

The study was conducted in Makka Al-Mukarama, in Saudi Arabia. One hundred and five participants who met the inclusion criteria were enrolled in the present study, ethical consent was obtained from each participant. The overall study methodology was approved by the Biomedical Ethics Committee, Faculty of Medicine, Umm AlQura University, Makkah, Kingdom of Saudi Arabia.

The study population was categorized into two groups of $H$. pylori positive cases and negative controls according to their results of Helicobacter pylori IgG antibodies. Subjects in both groups fill the structured questionnaire and subjected to anthropometric measurements and clinical investigations. Blood samples were drawn using standard collection procedures for evaluation of FBS, 2hr-PP blood sugar, HbA1c, Lipid profile and oxidized LDL.

Routine analysis was performed using a full automated chemistry analyzer (Humastar-80), from Human Diagnostics. The protocols were followed as manufacturer's guidance using the kits and controls of Human.

H. pylori antibodies were assessed using Abcam's Human Helicobacter pylori IgG ELISA Test kit. Briefly, 96-well plates have been pre-coated with Helicobacter pylori antigens, cognate antibodies were bound. Controls or test samples were added and incubated. Washing cycles then followed, a horseradish peroxidase (HRP) labeled anti-Human IgG conjugate was added which binds to the Helicobacter pylori-specific antibodies. TMB with HRP then produce a blue color product that changes to yellow after addition of an acid to stop the reaction. The density of the yellow color is directly proportional to the amount of Helicobacter pylori IgG in the samples.

Oxidized Low Density Lipoproteins was measured using Mercodia Oxidized LDL Competitive ELISA. The assay kit Manufactured by Mercodia AB, Sylveniusgatan 8A, SE-754 50 Uppsala, Sweden. This assay uses Holvoetet al monoclonal antibody, 4E6 specific for oxidatively modified LDL [13]. The 4E6 antibody is directed 
against a conformational change in the apoB-100 of LDL.

The principle is a competitive ELISA were the Oxidized LDL in the sample or standard competes with a fixed amount of oxidized LDL bound to the well for the binding to the specific antibodies 4E6. A washing step removes unreactive sample components, then the biotin-labeled antibody bound to the well is detected by HRPconjugated streptavidin. The bound conjugate is detected by reaction with 3, 3', 5, 5'-tetramethylbenzidine (TMB). The stop solution is added and a yellow color developed which can be read spectrophotometrically and the concentration of LDL determined using the curve generated from standards.

\section{Statistical Analysis}

The statistical analysis was done using Statistical Package for the Social Sciences (SPSS) for windows, version 20. Results were expressed as mean \pm SD. Descriptive statistics, the mean procedures and independent sample t-test were used to compare the different studied parameters between groups of $H$. pylori positive cases and negative controls, $\mathrm{P}<0.05$ was considered as statistically significant.

\section{Results}

Among the 105 Type-2 Diabetic patients, 48 cases (45.7\%) were diagnosed as $H$. pylori seropositive. The negative controls were 57 patients (54.3\%). The ages of participants in the present study ranges from 25 - 64 years, there is no significant difference in the mean age or mean BMI between the $H$. pylori negative and positive cases as shown in Table 1.

Glycemic control was similar in the two groups, HbA1c was higher in the H. pylori positive cases suggesting uncontrolled diabetes, but difference between the means was not statistically significant.

Total Cholesterol was found to be elevated in cases of positive $H$. pylori compared to negative controls $(\mathrm{P}<$ $0.001)$ and Triglycerides was found to be significantly elevated too in the same group $(\mathrm{P}<0.005)$.

While there was no significant difference in the levels of HDL-Cholesterol between the two groups, the mean LDL-Cholesterol was found to be significantly increased in cases of $H$. pylori positive compared to negative controls $(\mathrm{P}<0.001)$.

Oxidized LDL levels confirm the disturbance in lipid profile in the positive cases and found to be increased significantly in the $H$. pylori positive cases $(\mathrm{P}=0.001)$ compared to negative controls as shown in Table 1 . Lipid profile, when compared between the two groups shows marked dyslipidemia in cases of $H$. pylori, moreover, the increased atherogenic oxidized LDL in this group suggest a correlation between infection of $H$. pylori and disturbance in lipid metabolism.

\section{Discussion}

Since the discovery of Helicobacter pylori by Barry J. Marshall and Robin Warren in 1984, where they found that the bacteria was present in almost all subjects with active chronic gastritis, duodenal ulcer, or gastric ulcer

Table 1. Age, BMI, glucose tolerance and lipid profile in the studied groups.

\begin{tabular}{cccc}
\hline & H. pylori Positive $(\mathbf{N}=\mathbf{4 8})$ & H. pylori Negative $(\mathbf{N}=\mathbf{5 7})$ & P value \\
\hline Age Yrs. & $45.1 \pm 9.5$ & $45.4 \pm 9.6$ & 0.841 \\
BMI Kg/m ${ }^{2}$ & $29.2 \pm 4.3$ & $30.2 \pm 4.6$ & 0.273 \\
FBS mg/dl & $186 \pm 64$ & $192 \pm 60$ & 0.618 \\
2hr-pp mg/dl & $320 \pm 85$ & $295 \pm 72$ & 0.165 \\
HbA1c \% & $8.1 \pm 2.1$ & $7.8 \pm 1.8$ & 0.419 \\
Cholesterol mg/dl & $288 \pm 76$ & $221 \pm 47$ & $<0.001$ \\
Triglycerides mg/dl & $257 \pm 180$ & $179 \pm 88$ & 0.005 \\
HDL-C mg/dl & $53.5 \pm 16.7$ & $49.2 \pm 16.5$ & 0.187 \\
LDL-C mg/dl & $168.6 \pm 43.6$ & $133.5 \pm 38.1$ & $<0.001$ \\
Ox-LDL U/L & $142.3 \pm 21.2$ & $125.8 \pm 28.8$ & 0.001 \\
\hline
\end{tabular}


and concluded that it may be an important etiological factor of these diseases, since then and the bulk of research was focused on the effect of the bacteria on the gastro-intestinal region [14].

Current data suggestan extra-gastric role of $H$. pylori, there is evidence that $H$. pylori infection is associated with increased incidence of Type-2 Diabetes mellitus [15], and may be a major contributing factor for development of insulin resistance [16], long-term diabetes complications, and cardiovascular risk factors [17], however there is limited data supporting this controversial association [18] and some previous studies did not find it [19]-[21]. Moreover, while some studies look at $H$. pylori infection as a risk factor for diabetes an eradication can improve the mean glycated hemoglobin in diabetic patients [22], others interpret the association as susceptibility of diabetic patients to the infection [23].

The present study investigates the disturbance in lipid profile in Type-2 diabetic patients with and without $H$. pylori infection. The findings suggest marked dyslipidemia and a significant increase in Total Cholesterol levels in seropositive cases as shown in Figure 1, these findings are consistent with many previous studies that report elevated serum LDL cholesterol levels in elder population with $H$. pylori [24]. Cardiovascular complications of diabetes mellitus are caused by dyslipidemia, in particular the increased LDL and oxidized LDL [25]. H. pylori infection was reported to be more prevalent in CAD positive patients [12], this may be due to the disturbance in lipid metabolism associated with $H$. pylori infection. Diabetic patients with positive $H$. pylori score high in triglyceride levels with a significant difference when compared with their negative peers in the present study. Although most of the previous studies focus on total cholesterol and LDL-C as parameters of cardio-metabolic disorders, but the significant increase in seropositive subjects may support the hypothesis that the dyslipidemia observed in this group have a strong association with the H. pylori infection.

Oxidized LDL is considered as a biomarker of cardiovascular diseases [26]. In the present study the levels of ox-LDL was significantly higher in $H$. pylori positive diabetic patients as shown in Figure 2, eradication of the infection was supposed to be a promising strategy to control lipid profile as well as other metabolic parameters [5].

The gastric inflammation caused by $H$. pylori infection was proposed as a risk factor for insulin resistance and cardiovascular diseases in Type-2 Diabetes [18]. The disturbance in lipid profile which is associated with $H$. $p y-$ lori infection seems to play a significant role in the mechanism of these diabetic complications, considering the different metabolic roles of $H$. pylori especially in diabetic patients may help in treatment and prevention of many of these complications.

Before Warren and Marshall announced their findings about the role of $H$. pylori in gastritis and duodenal ulcer, it was a common and long-standing belief in medical teaching and practice that the major causes of peptic ulcer disease were stress and lifestyle factors. Soon after, it was clear with strong evidences that $H$. pylori, causes more than $90 \%$ of duodenal ulcers and up to $80 \%$ of gastric ulcers [27]. Now a growing evidence suggests that there are many extra-intestinal manifestations of $H$. pylori infection, and if validated, this may provide a better understanding and monitoring of many of the pathologies associated with H. pylori.

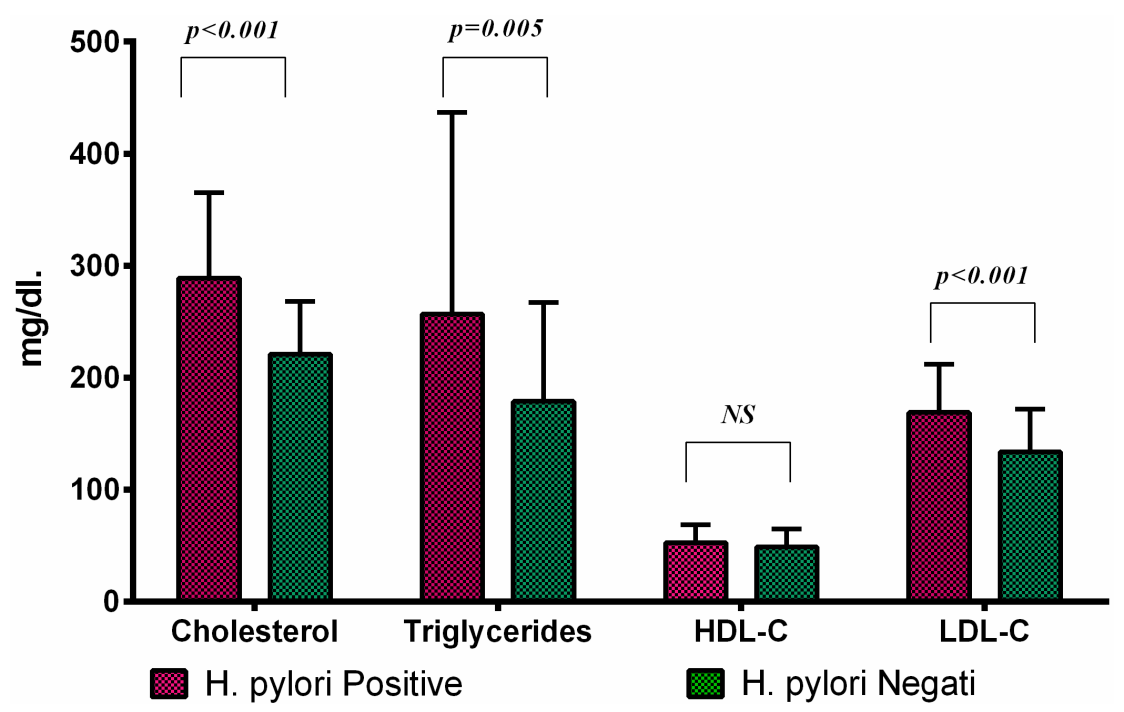

Figure 1. Cholesterol, triglyceride, HDL-C, and LDL-C in H. pylori positive and H. pylori negative subjects. 


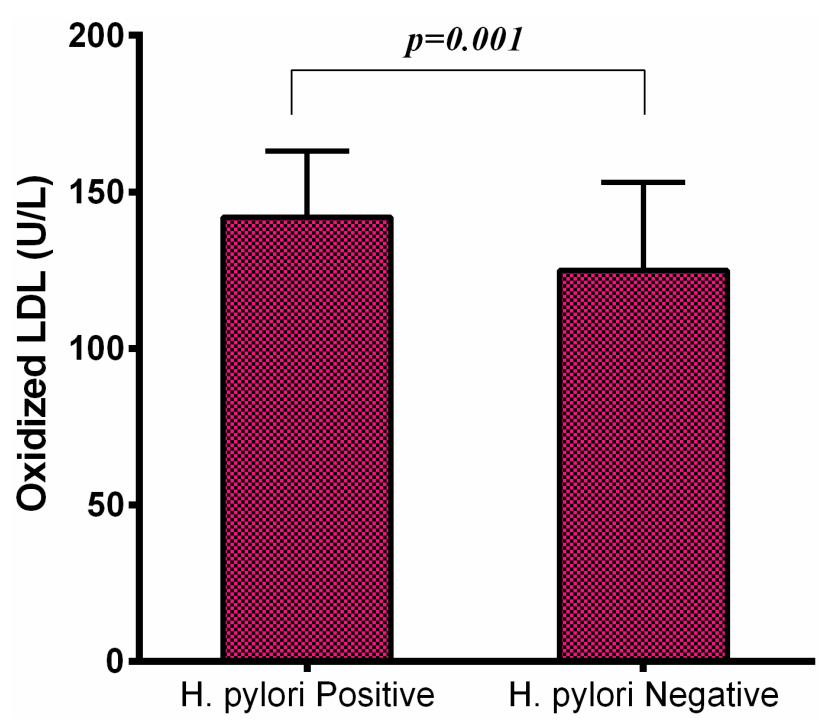

Figure 2. Oxidized LDL in H. pylori positive band H. pylori negative subjects.

\section{Conclusion}

Infection with $H$. pylori is associated with increased levels of Total Cholesterol, Triglycerides, LDL-C and oxidized LDL in Type 2 Diabetic patients. And eradication of the pathogen may represent a useful tool for monitoring dyslipidemia in infected diabetic subjects.

\section{Acknowledgements}

This research project was supported and funded by a grant from the Institute of Scientific Research and Revival of Islamic Culture, Umm Al-Qura University, Makkah, Kingdom of Saudi Arabia.

\section{Conflict of Interests}

The authors declare that there is no conflict of interests regarding the publication of this paper.

\section{Author Contributions}

MHM: design of the study, interpretation of biochemical laboratory investigations, and editing, styling, writing, and revising of the main manuscript text. WMN: design of the study, interpretation of biochemical laboratory investigations, preparation of tables and figures, and editing, styling, writing, and revising of the main manuscript text. ATB: biochemical laboratory investigations, statistical analysis, preparation of tables and figures, and editing, styling, writing, and revising of the main manuscript text. All authors read and approved the final manuscript.

\section{References}

[1] Testerman, T.L. and Morris, J. (2014) Beyond the Stomach: An Updated View of Helicobacter pylori Pathogenesis, Diagnosis, and Treatment. World Journal of Gastroenterology, 20, 12781-12808. http://dx.doi.org/10.3748/wjg.v20.i36.12781

[2] Nagy, P., Johansson, S. and Molloy-Bland, M. (2016) Systematic Review of Time Trends in the Prevalence of Helicobacter pylori Infection in China and the USA. Gut Pathogens, 8, 8. http://dx.doi.org/10.1186/s13099-016-0091-7

[3] Peleteiro, B., Bastos, A., Ferro, A. and Lunet, N. (2014) Prevalence of Helicobacter pylori Infection Worldwide: A Systematic Review of Studies with National Coverage. Digestive Diseases and Sciences, 59, 1698-1709. http://dx.doi.org/10.1007/s10620-014-3063-0

[4] Sapmaz, F., et al. (2016) The Impact of Helicobacter pylori Eradication on Serum Hepcidin-25 Level and Iron Parameters in Patients with Iron Deficiency Anemia. Wiener klinische Wochenschrift, 128, 335-340. http://dx.doi.org/10.1007/s00508-016-0961-5 
[5] Buzas, G.M. (2014) Metabolic Consequences of Helicobacter Pylori Infection and Eradication. World Journal of Gastroenterology, 20, 5226-5234. http://dx.doi.org/10.3748/wjg.v20.i18.5226

[6] He, C., Yang, Z. and Lu, N.H. (2014) Helicobacter pylori Infection and Diabetes: Is It a Myth or Fact? World Journal of Gastroenterology, 20, 4607-4617. http://dx.doi.org/10.3748/wjg.v20.i16.4607

[7] Dai, Y.N., et al. (2015) Is Helicobacter pylori Infection Associated with Glycemic Control in Diabetics? World Journal of Gastroenterology, 21, 5407-5416. http://dx.doi.org/10.3748/wjg.v21.i17.5407

[8] Wong, F., Rayner-Hartley, E. and Byrne, M.F. (2014) Extraintestinal Manifestations of Helicobacter pylori: A Concise review. World Journal of Gastroenterology, 20, 11950-11961. http://dx.doi.org/10.3748/wjg.v20.i34.11950

[9] Chmiela, M., Gajewski, A. and Rudnicka, K. (2015) Helicobacter pylori vs Coronary Heart Disease-Searching for Connections. World Journal of Cardiology, 7, 187-203.

[10] Sharma, V. and Aggarwal, A. (2015) Helicobacter pylori: Does It Add to Risk of Coronary Artery Disease. World Journal of Cardiology, 7, 19-25. http://dx.doi.org/10.4330/wjc.v7.i1.19

[11] Aroner, S.A., et al. (2016) Fetuin-A, Glycemic Status, and Risk of Cardiovascular Disease: The Multi-Ethnic Study of Atherosclerosis. Atherosclerosis, 248, 224-229. http://dx.doi.org/10.1016/j.atherosclerosis.2016.03.029

[12] Vafaeimanesh, J., et al. (2014) Association of Helicobacter pylori Infection with Coronary Artery Disease: Is Helicobacter pylori a Risk Factor? Scientific World Journal, 2014, Article ID: 516354.

[13] Holvoet, P., et al. (2006) Analytical Performance and Diagnostic Accuracy of Immunometric Assays for the Measurement of Circulating Oxidized LDL. Clinical Chemistry, 52, 760-764. http://dx.doi.org/10.1373/clinchem.2005.064337

[14] Marshall, B.J. and Warren, J.R. (1984) Unidentified Curved Bacilli in the Stomach of Patients with Gastritis and Peptic Ulceration. The Lancet, 323, 1311-1315. http://dx.doi.org/10.1016/S0140-6736(84)91816-6

[15] Jeon, C.Y., et al. (2012) Helicobacter pylori Infection Is Associated with an Increased Rate of Diabetes. Diabetes Care, 35, 520-525. http://dx.doi.org/10.2337/dc11-1043

[16] Chen, L.W., et al. (2015) Helicobacter pylori Infection Increases Insulin Resistance and Metabolic Syndrome in Residents Younger than 50 Years Old: A Community-Based Study. PLoS One, 10, e0128671. http://dx.doi.org/10.1371/journal.pone.0128671

[17] Hamed, S.A., et al. (2008) Vascular Risks and Complications in Diabetes Mellitus: The Role of Helicobacter pylori Infection. Journal of Stroke \& Cerebrovascular Diseases, 17, 86-94. http://dx.doi.org/10.1016/j.jstrokecerebrovasdis.2007.10.006

[18] Bahadoran, Z., et al. (2015) Helicobacter pylori Stool Antigen Levels and Serological Biomarkers of Gastric Inflammation Are Associated with Cardio-Metabolic Risk Factors in Type 2 Diabetic Patients. Endocrinology and Metabolism (Seoul), 30, 280-287. http://dx.doi.org/10.3803/EnM.2015.30.3.280

[19] Oluyemi, A., et al. (2012) Prevalence of a Marker of Active Helicobacter pylori Infection among Patients with Type 2 Diabetes Mellitus in Lagos, Nigeria. BMC Research Notes, 5, 284. http://dx.doi.org/10.1186/1756-0500-5-284

[20] Malamug, L.R., et al. (2014) The Role of Helicobacter pylori Seropositivity in Insulin Sensitivity, Beta Cell Function, and Abnormal Glucose Tolerance. Scientifica (Cairo), 2014, Article ID: 870165.

[21] Xia, H.H., et al. (2001) Helicobacter pylori Infection Is Not Associated with Diabetes Mellitus, Nor with Upper Gastrointestinal Symptoms in Diabetes Mellitus. The American Journal of Gastroenterology, 96, 1039-1046. http://dx.doi.org/10.1111/j.1572-0241.2001.03604.x

[22] Zojaji, H., et al. (2013) The Effect of the Treatment of Helicobacter pylori Infection on the Glycemic Control in Type 2 Diabetes Mellitus. Gastroenterology and Hepatology from Bed to Bench, 6, 36-40.

[23] Devrajani, B.R., et al. (2010) Type 2 Diabetes Mellitus: A Risk Factor for Helicobacter pylori Infection: A Hospital Based Case-Control Study. International Journal of Diabetes in Developing Countries, 30, 22-26. http://dx.doi.org/10.4103/0973-3930.60008

[24] Kim, H.L., et al. (2011) Helicobacter pylori Infection Is Associated with Elevated Low Density Lipoprotein Cholesterol Levels in Elderly Koreans. Journal of Korean Medical Science, 26, 654-658. http://dx.doi.org/10.3346/jkms.2011.26.5.654

[25] Harmon, M.E., et al. (2016) Associations of Circulating Oxidized LDL and Conventional Biomarkers of Cardiovascular Disease in a Cross-Sectional Study of the Navajo Population. PLoS One, 11, e0143102. http://dx.doi.org/10.1371/journal.pone.0143102

[26] Trpkovic, A., et al. (2015) Oxidized Low-Density Lipoprotein as a Biomarker of Cardiovascular Diseases. Critical Reviews in Clinical Laboratory Sciences, 52, 70-85. http://dx.doi.org/10.3109/10408363.2014.992063

[27] Ahmed, N. (2005) 23 Years of the Discovery of Helicobacter pylori: Is the Debate over? Annals of Clinical Microbiology and Antimicrobials, 4, 17. http://dx.doi.org/10.1186/1476-0711-4-17 


\section{Submit or recommend next manuscript to SCIRP and we will provide best service for you:}

Accepting pre-submission inquiries through Email, Facebook, Linkedin, Twitter, etc A wide selection of journals (inclusive of 9 subjects, more than 200 journals)

Providing a 24-hour high-quality service

User-friendly online submission system

Fair and swift peer-review system

Efficient typesetting and proofreading procedure

Display of the result of downloads and visits, as well as the number of cited articles

Maximum dissemination of your research work

Submit your manuscript at: http://papersubmission.scirp.org/ 